\title{
SENSOR FAULT ACCOMMODATION : APPLICATION TO AN ACTIVATED SLUDGE PROCESS.
}

\author{
C. Aubrun, C. Leick \\ Centre de Recherche en Automatique de Nancy, \\ CNRS URA 821, Université Henri Poincaré Nancy I, 54500 Vandøuvre, France \\ Fax : $33(0) 383684462$ \\ Email : christophe.aubrun@cran.uhp-nancy.fr
}

\begin{abstract}
The effect of changes in operating conditions due to fault occurrences can alter performances in industrial processes. One way of preventing performances from degradation is to modify the control law in order to maintain the functioning of the process under acceptable operating conditions. In this paper, a Fault Tolerant Control of an Activated Sludge Process is presented. Free fault data are reconstructed from the faulty one by mean of a Recursive Principal Component Analysis and the control law is designed on the basis of the reconstructed data. Copyright ${ }^{\circ} 2005$ IFAC
\end{abstract}

Keywords : Fault accommodation; Recursive principal component analysis; Adaptive process monitoring; Activated sludge process

\section{INTRODUCTION}

In the past decades, studies concerning Fault Tolerant Control (FTC) were mainly related to flight control systems for obvious safety reasons (Rausch, 1994; Passino et al., 1995). Recently, they were directed towards industrial processes in order to improve the reliability of the production and quality of products. An extensive survey on FTC can be found in (Patton, 1997) and (Blanke et al., (2000).

Different approaches are possible according to the severity of the failure. The goal of the FTC is to determine a new control law that takes the degraded system parameters into account and drives the system to a new operating point preserving the main control performances (stability, accuracy,...) (Gao and Antsalkis, 1992; Looze et a.,l 1985; Bodson and Groszkiewicz, 1997; Aubrun, et al., 1993; Noura et al., 2000).

A natural way of coping with the FTC problem is to modify the control law parameters according to an on-line identification of the system parameters when a fault occurs. The faults effects appear as parameter changes, which are identified on line, and the control law is accommodated automatically based on these new parameters (Rausch, 1995).

In the approach proposed by (Bonivento et al., 2004) FTC consists in designing a controller embedding an internal model of the faults. Following this method, the faults effects are intrinsically compensated. This approach does not require an explicit Fault Detection an Isolation step.

Another way of achieving accommodation relies on the reconstructed data. Faults are compensated via an appropriate control law triggered according to the diagnosis of the system before it propagates to other elements of the process. Fig. 1 shows the general scheme of the FTC procedure. A performance evaluation module, analyses the actual operating conditions. In case of fault occurrence, the accommodation module determines new set point 
or/and modify the controller structure accordingly to the fault severity.

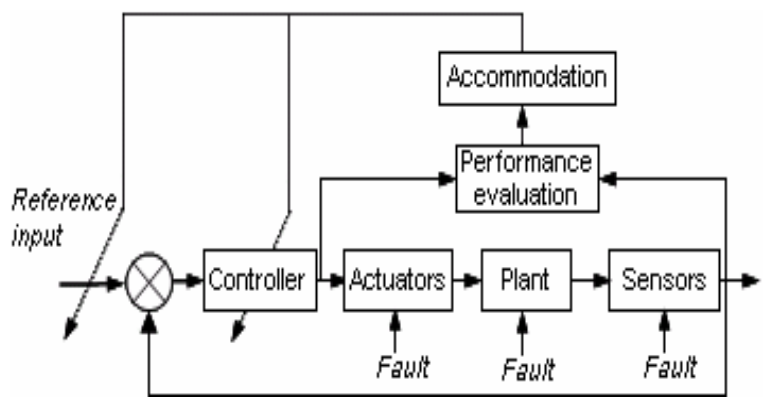

Fig 1. Scheme of the FTC

This paper presents recent developments within IFATIS project (EU-IST-2001-32122). It focuses on the design of a sensor failure accommodation module for Yorkshire Water ASP. FTC is particularly important to processes where continuity of production or operation is paramount. Aeration process commonly used to treat wastewater falls within this category and should benefit from the application of FTC approaches. The key features of control of this type of plant is the measurement of Dissolved Oxygen (DO) at key points along a process train, and forced delivery of air to maintain a specific DO value under changing process conditions. The performance of biological systems is typically improved by minimising disturbances to their environmental parameters. In general, achieving and maintaining DO levels is the key control objective with ambient temperature being a natural, but slowly varying disturbance. Low levels of sensor redundancy and a severe instrument environment characterize the operation of this type of plant. In ASP the performances and operations are measured continuously. Usually the number of variable is high requiring a methodology to extract and structure useful information from sets of data. For this purpose, Principal Component Analysis (PCA) has found wide application in process monitoring. However, it is clear that this method is not suitable for monitoring processes that display non-stationary behaviour. An efficient way to circumvent this problem is to update the model recursively (Rozen, 1998; Dayal and Macgregor, 1997). (Li et al., 1999) propose a RPCA to adapt for normal process changes to reduce false alarm. The algorithm calculate recursively the correlation matrix with time varying mean and variance. Once a model describing the normal process operating condition faulty data are reconstructed and then, are replaced with best reconstruction in order to maintain control system on line (Qin and Li, 1999).

The paper is organized as follows. After a brief description of the Benchmark, the paper presents in section 3 the Recursive Principal Component Analysis (RPCA) approach. The adaptation mechanism is described. In section 4 the method is applied to accommodate the system to a DO sensor failure. Finally, in section 5 the benchmark application is detailed and different faults scenario are studied.

\section{BENCHMARK DESCRIPTION}

The process is the aeration of industrial effluent using mechanical aeration followed by tapered aeration using air from a pressurised ring-main. The effluent is split into parallel lanes and each lane is divided into three aeration zones. There are a total of 4 DO instruments installed to measure the oxygen concentrations. One is located in the mechanical zone and one in each of the three zones. Fig. 2, presented in appendix, shows the basic layout of one lane.

The air used in the process is provided by a combination of up to 4 blowers and the air flow rate for each of the three zones (1,2 and 3$)$ is controlled by local PLC. In each of the tapered aeration zones that follow after the mechanical aeration zone, there are control valves that modulate the flow of air into the effluent to maintain the DO in that zone at the desired value. The control is achieved by the mean of PI controller. The position of the valve (\%) is adjusted to supply a volume of air to the corresponding zone.

A benchmark has been developed and implemented using SIMULINK/MATLAB in order to evaluate FTC strategy. The initialisation procedure for the states and parameters values are determined in such a way that that it faithfully reproduces the real behaviour of the ASP in terms of internal dynamics, of time varying parameters, of structural nonlinearities. The following measurements are available to model the process:

- DO mech: DO measurement in mechanical zone

- DO $\mathrm{Zi}$ : DO measurement in zone $\mathrm{i}(\mathrm{i}=1,2,3)$

- F : Influent flow rate

- V_i : Valve opening in zone $\mathrm{i}(\mathrm{i}=1,2,3)$

The suspended solids concentration may display large and fast changes in the measurement. It is observed that the probes may read low due to built up of rag on the sensor. This will result in an unexpected increase of the control signal on the PI controller in the corresponding zone leading to a high value of $\mathrm{DO}$.

\section{RPCA FOR DATA RECONSTRUCTION}

In PCA we are concerned with finding the latent vector space that explains the greatest amount of variability in a single matrix of data.

Let us consider, $\mathrm{x}(\mathrm{k})$, a set of $\mathrm{m}$ variable. Assume that the process data are given in a matrix X ( $\mathrm{n} \mathrm{x} \mathrm{m)}$ 
where $\mathrm{n}$ represents the number of samples.. $\mathrm{X}$ is approximated as

$$
\mathrm{X} \cong \hat{\mathrm{X}}=\sum_{\mathrm{i}=1}^{\mathrm{k}} \mathrm{t}_{\mathrm{i}} \mathrm{p}_{\mathrm{i}}^{\mathrm{T}}
$$

where $p_{i}$ are the first $k$ largest eigenvectors $\left(p_{1} \ldots p_{k}\right)$ of the covariance matrix of $\mathrm{X}$ and the scores $t_{i}$ are linear combinations of the process measurement and are defined by

$$
\mathrm{t}_{\mathrm{i}}=\mathrm{Xp}
$$

By applying PCA on matrix $\mathrm{X}$, the information contained in $\mathrm{X}$ can be summarized by a lower dimensional score space defined by the principal components and reduce the dimension of the analysis space. Then, identification of the process model by PCA consists in determining eigenvalues and eigenvectors of matrix $\mathrm{X}^{\mathrm{T}} \mathrm{X} . \quad \mathrm{x}(\mathrm{k}) \in \mathfrak{R}^{\mathrm{n}}$ is decomposed into

$$
x(k)=P P^{T} x(k)+\widetilde{P} \widetilde{P}^{T} x(k)
$$

where $\mathrm{P} \in \mathfrak{R}^{\mathrm{n} \times 1}$ are eigenvectors corresponding to the principal eigenvalues $\lambda_{1} \geq, \ldots, \geq \lambda_{\ell}$ of the correlation matrix of $\mathrm{x}(\mathrm{k})$ and $\widetilde{\mathrm{P}}$ are eigenvectors corresponding to the remaining eigenvalues $\lambda_{\ell+1} \geq, \ldots, \geq \lambda_{\mathrm{n}}$. Then, the reconstructed part of $x(k)$ is given by the following expression

$$
\hat{x}(\mathrm{k})=P P^{T} x(k)=C x(k)
$$

And the residual part of $x(k)$ is given by

$$
\widetilde{\mathrm{x}}(\mathrm{k})=\widetilde{\mathrm{P}} \widetilde{\mathrm{P}}^{\mathrm{T}} \mathrm{x}(\mathrm{k})=(\mathrm{I}-\mathrm{C}) \mathrm{x}(\mathrm{k})=\widetilde{\mathrm{C}} \mathrm{x}(\mathrm{k})
$$

Consider a set of data in which variable $x_{i}$ is supposed to be faulty. $\mathrm{x}_{\mathrm{i}}(\mathrm{k})$ is arbitrarily set to zero.

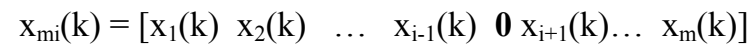

and let e be the reconstruction error defined as

$$
\mathrm{e}(\mathrm{k})=\mathrm{x}(\mathrm{k})-\hat{\mathrm{x}}(\mathrm{k})
$$

which gives

$$
\mathrm{e}(\mathrm{k})=\mathrm{x}_{\mathrm{mi}}(\mathrm{k}) \widetilde{\mathrm{C}}+\mathrm{x}_{\mathrm{i}}(\mathrm{k}) \widetilde{\mathrm{C}}_{\mathrm{i}}
$$

Then the faulty value can be reconstructed as follows

$$
x_{i}(k)=-\frac{x_{m i}(k) \widetilde{C} \widetilde{C}_{i}^{T}}{\widetilde{C} \widetilde{\mathrm{C}}_{i}^{T}}
$$

The detection index is the Squared Prediction Error (SPE) defined by

$$
\mathrm{d}(\mathrm{k})=\mathrm{e}(\mathrm{k})^{\mathrm{T}} \mathrm{e}(\mathrm{k})
$$

PCA assumes that data are stationary which is usually not the case in ASP monitoring. This limitation can be overcome by use of an adaptive PCA. The PCA model is continuously updated using an exponential memory. Then, the model has to adapt to slow modification of the process operating condition. At each iteration the covariance matrix is updated recursively as follows (Dayal and MacGregor 1997)

$$
\mathrm{X}^{\mathrm{T}} \mathrm{X}(\mathrm{k})=\alpha \mathrm{X}^{\mathrm{T}} \mathrm{X}(\mathrm{k}-1)+(\alpha-1) \mathrm{x}(\mathrm{k})^{\mathrm{T}} \mathrm{x}(\mathrm{k})
$$

Where $\alpha$ is a forgetting factor. The value of $\alpha$ is adjusted according to the dynamics of the system.

\section{DO SENSOR FAULT ACCOMMODATION}

The scheme of the DO sensor fault accommodation is illustrated in Fig 3.

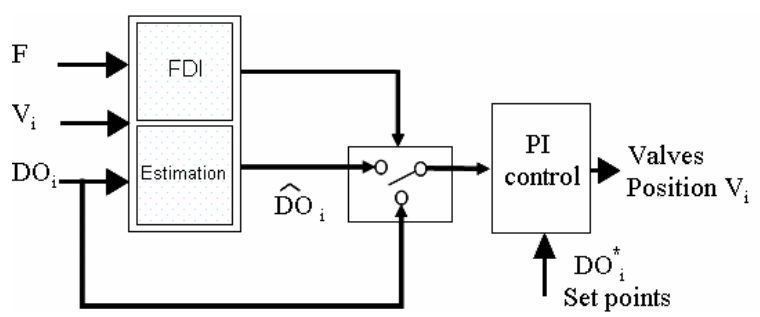

Fig 3. Sensor fault accommodation mechanism

The decision mechanism triggers an alarm and the faulty measurement is automatically replaced by the free fault estimation computed on the basis of the updated model.

The fault detection and isolation is based on the partial SPE index principle (Kourti and MacGregor 1996 ; Harkat et al., 2000). The principle of partial SPE is represented in Fig. 4.

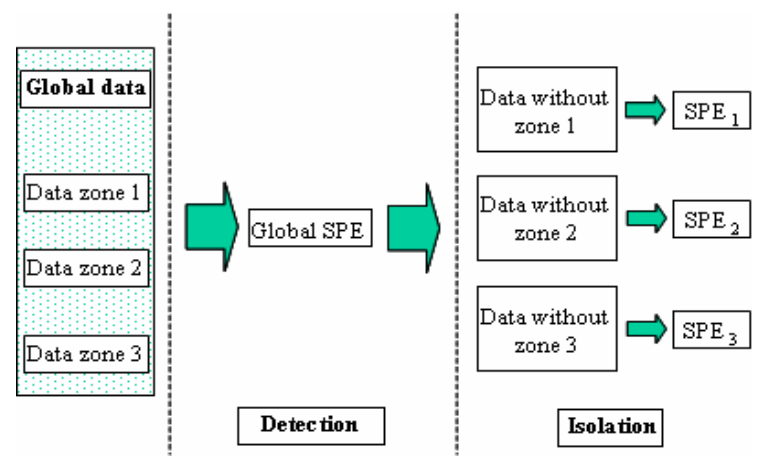

Fig. 4. Fault isolation by partial SPE

The PCA approach combined with appropriate index allows detection but cannot isolate the fault. It can be achieved by considering separately the contribution of each data on SPE index. The partial SPE is built from the whole data vector after removing data related to the different zone. The contribution of each zone on the detection index is quantified by mean of the partial SPE. The lower is the SPE value of a 
zone, the higher is the probability that fault occurred in that zone.

\section{BENCHMARK APPLICATION}

The fault accommodation procedure described above was applied on the benchmark of the ASP.

- The sample period is 5 minutes and the duration of the experiment is 14 days.

- The data vector is built as follows:

$\mathrm{X}=\left[\mathrm{V} \_1 \mathrm{~V} \_2 \mathrm{~V} \_3 \mathrm{~F}\right.$ DO_Z3 DO_Z2 DO_Z1]

- The faults scenarios are:

- Fault 1 : abrupt bias on DO probe measurement in zone 2 between instant 6.5 and 7.5 (Bias magnitude of $0.5 \mathrm{mg} / \mathrm{l}$ )

- Fault 2 : Complete breakdown of DO probe in zone 3 between instant 8 and 8.5

- Fault 3 slow drift of DO probe measurement in zone 1 between instant 9 and 11 (Drift $+0.4 \mathrm{mg} / \mathrm{l}$ per day due to probe soiling mode).

The investigations were performed in closed loop configuration and following two cases study :

a) Faulty data with no accommodation algorithm

b) Faulty data with accommodation algorithm

The diagnostic and reconstruction algorithm was designed and tested with data containing the three faults at the same time.

\subsection{Fault detection results}

The graph presented in Fig. 5 shows the evolution of the global SPE index during 14 days.

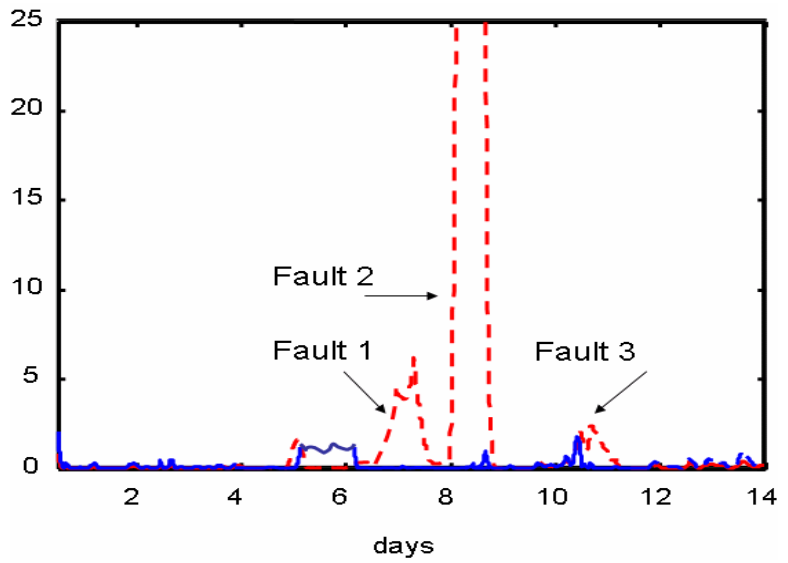

Fig. 5. Global SPE index (---without reconstruction;- with reconstruction)

As the global SPE index is computed on the basis of all measurement it does not allow to isolate the fault. The dashed line (SPE index without data reconstruction) indicates clearly the occurrence of fault 1 and 2 meanwhile the detection is not very effective in case of fault 3 .

The partial SPE is applied on raw data set. Results are summarised in table1.
Table 1. Fault contribution for each zone

\begin{tabular}{lcllll}
\hline Fault & $\begin{array}{c}\text { Detection } \\
\text { time }\end{array}$ & End & SPE_3 & SPE_2 & SPE_1 \\
Scenario & 6.5 & 7.6 & 1.74 & 0.64 & 2.8 \\
Fault 1 & 8.1 & 8.8 & 0.03 & 51.69 & 22.10 \\
Fault 2 & 8.1 & 10.9 & 2.58 & 1.04 & 0.08 \\
Fault 3 & 10.4 & 10.9 & \\
\hline
\end{tabular}

\subsection{Sensor fault accommodation}

The fault accommodation is conducted after the fault has been detected. Fig 6 shows the fault identification result for the DO measurement in zone 1. The plot of the DO values are presented in Fig 6 . Table 1 gives detection time 10.39. This delay seems to be reasonable as the fault is a drift. The doted line showed in Fig. 6 (non compensated fault) exhibits a large magnitude peak at instant 10.4 meanwhile the reconstructed data follows quite fairly the fault free values.

The accommodation procedure prevents the system from fault propagation. Dashed line at time index 8 exhibits peaks due to the compensated fault occurring in zone 2 .

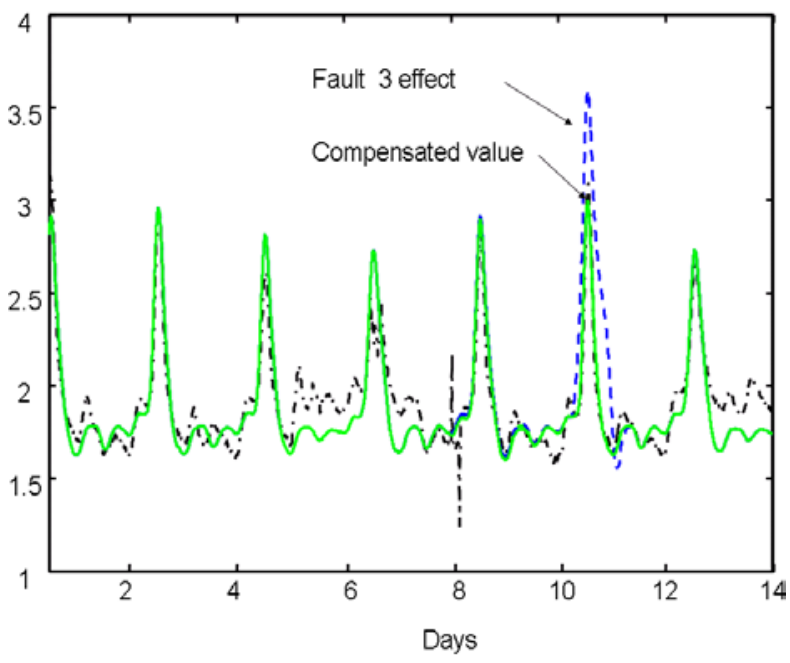

Fig. 6. reconstructed DO measurement

\section{CONCLUSIONS}

A fault complete sensor fault accommodation scheme is proposed based on RPCA approach to reconstruct faulty data. The fault is detected, isolated and replaced by its best estimation computed by the RPCA algorithm. Three type sensor fault, bias, drift, complete failure are considered. The detection and isolation, based on a partial squared prediction error index, exhibits good performances for the bias and complete failure. The adaptive mechanism of PCA introduce a delay in the detection for the drift. Nevertheless, when the fault is detected, the accommodation procedure is effective. 


\section{ACKNOWLEDGEMENTS}

The authors wish to acknowledge the support that Yorkshire Water staff has given in the development given in the development of this project.

\section{REFERENCES}

Aubrun, C., Sauter, D., Noura N., and Robert, M. (1993). Fault diagnosis and reconfiguration of systems using fuzzy logic: application to a thermal plant. International Journal of System Science 24(10), 1945-1954.

Bodson, M., and Groszkiewicz, J. (1997). Multivariable Adaptive Algorithms for Reconfigurable Flight Control. IEEE Transactions on Control Systems Technology 5(2), 217-229.

Bonivento, C., Isidori, A., Marconi L., Paoli, A. (2004) Implicit fault tolerant control : application to an induction motors. Automatica, (40)355-371

Blanke, M., Frei, C.W., Krauss, F., Patton, R.J., Staroswiecki, M. (2000). What is fault tolerant control? IFAC Symposium on Fault Detection, Supervision and Safety for Technical Processes, SAFEPROCESS'2000 Budapest, June 14-16.

Dayal, B., and MacGregor J. (1997) Recursive exponentially weighted PLS and its applications to adaptive control and prediction. J. Proc. Cont. (7) 3 169-179

Gao, Z., and Antsalkis, P.J. (1992). Reconfigurable control system design via perfect model following. International Journal of Control, 56(4), 783-798.

Harkat, M.F., Mourot G., Ragot J. (2000). Sensor Failure Detection of Air Quality Monitoring Network. IFAC Symposium on Fault Detection, Supervision and Safety for Technical Processes, SAFEPROCESS'2000 Budapest, June 14-16
Kourti T., MacGregor J.F. (1996). Recent Developments in Multivariable SPC Methods for Monitoring and Diagnosis Process and Product Performance. Jourbal Of Quality Technology, (28)4, pp 409-428

Li, W., Yue, H., Valle-Cervantes, S. and S. Qin J. (1999), Recursive PCA for adaptive process monitoring, Journal of process control (10), 5, October 2000, Pages 471-486

Looze, D.P., Weiss, J.L., Eterno, J.S., \& Barett, N.M. (1985) An automatic redesign approach for restructurable control systems. IEEE Control Systems Magazine 5(2), 16-22.

Noura, H., Theilliol, D., \& Sauter, D. (2000). Actuator fault-tolerant control design : demonstration on a three-tank-system. International Journal of System Science 31(9), 1143-1155.

Passino, K.M., Laukonen, E.G., \& Yurkovitch, S. (1995). Expert supervision of fuzzy learning systems for fault tolerant aircraft control. Proc. IEEE 83(3), 466-483.

Patton, R.J. (1997). Fault tolerant control: The 1997 situation. Proc. SAFEPROCESS' 97 (pp 10331055). Hull, UK.

Qin, J., and Weihua Li (1999) Detection, Identification, and Reconstruction of Faulty Sensors with Maximized Sensitivity - AIChE Journal, Vol(45) 9 , Pages 1963-1976

Rausch, H. (1994) Intelligent fault diagnosis and control reconfiguration. IEEE Control System Magazine 14(3), 6-12.

Rausch, H. (1995) Autonomous control reconfiguration, IEEE Control System Magazine 15(6), 37-49.

Rozen, C. (1998) Monitoring waste water treatment systems, Tech Lic. Thesis, Department of Industrial Electrical Engineering and Automation, Lund University, Lund, Sweden

\section{APPENDIX}

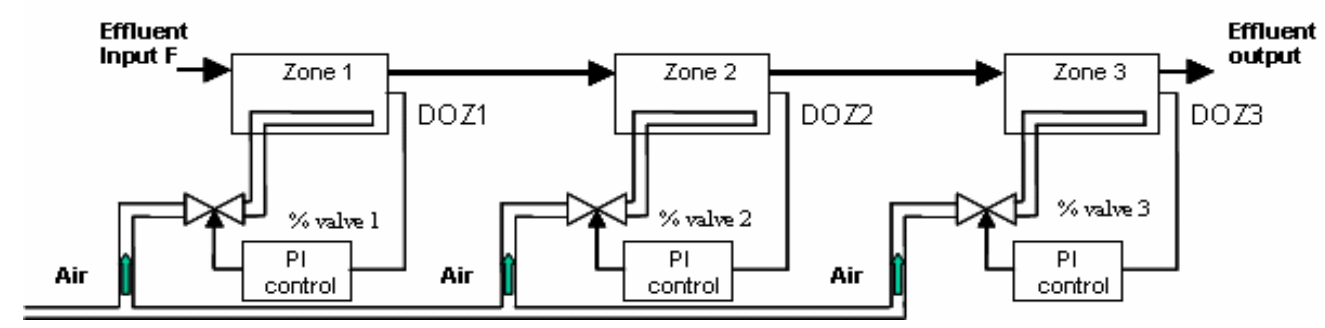

Fig. 2. Basic layout of one lane. 